J. Japan. Soc. Hort, Sci. 49(1) : 93-101. 1980.

\title{
Salt Tolerance of Muskmelons in Sand and Nutrient Solution Cultures
}

\author{
Akira Nukaya, Masao Masui and Akira Ishida \\ College of Agriculture, Shizuoka University, Ohya, Shizuoka 422
}

\begin{abstract}
Summary
Experiments were conducted to determine the effect of diluted sea water on the germination of muskmelon seeds, the seedling growth, and the growth in sand and nutrient solution cultures. The germination rate was not significantly different from 0 to $5,000 \mathrm{ppm} \mathrm{Cl}$ and decreased at $6,000 \mathrm{ppm} \mathrm{Cl} 7$ days after starting the germination test. Fresh weight of whole seedlings grown in sand for 16 days after germination decreased as sea water concentrations increasaed. Whole plant and fruit fresh weight in sand and nutrient solution cultures decreased with increasing sea water concentrations. The salt injury was observed at 250 to $1,000 \mathrm{ppm} \mathrm{Cl}$ in sand culture and at 3,000 to $5,000 \mathrm{ppm} \mathrm{Cl}$ in nutrient solution culture. The degree of injury became more severe with increasing sea water concentrations. Osmotic potential of leaves, and transpiration rate and amount decreased as sea water concentrations increased. Root osmotic potential was higher than that of leaves. Content of $\mathrm{Na}$ in leaves and $\mathrm{Cl}$ in each plant part increased with increasing sea water concentrations. In nutrient solution culture the $\mathrm{Na}$ and $\mathrm{Cl}$ accumulated most in stem, followed by roots and fruit, and accumulated least in leaves. $\mathrm{Na}$ and $\mathrm{Cl}$ content in leaves was markedly high at $5,000 \mathrm{ppm} \mathrm{Cl}$ in nutrient solution culture because fruit did not set due to poor growth.
\end{abstract}

\section{Introduction}

It is obvious that the salinity $\left(\mathrm{Cl}^{-}, \mathrm{Na}^{+}\right.$, $\mathrm{SO}_{4}{ }^{2-}, \mathrm{K}^{+}, \mathrm{Mg}^{2+}$ and $\mathrm{Ca}^{2+}$ ) in the well water of greenhouse growers in the Shizuoka Prefecture is relatively high and originated from sea water (13). Therefore, an experiment on muskmelons (Cucumis melo L.cv. Earl's Favourite) was made in soil culture by applying diluted sea water solutions. From this experiment the fresh weight of fruit was found to decrease with increasing sea water concentrations from 250 to $1,000 \mathrm{ppm}$ $\mathrm{Cl}$ (18). It is well known that the salt tolerance of plants, the salt injury symptoms and the degree of injury vary with the culture medium, the amount or kinds of excess salt, variety of crops, climate and so on. Because little information was found on salt tolerance of muskmelons $(18,22,24)$ the present experiments were conducted to determine the effect of diluted sea water on the growth of muskmelons in sand and nutrient Received for publication October 20, 1979. solution cultures. In addition, the effect of diluted sea water on seedling growth in sand culture, and germination and root elongation of the seeds were examined.

\section{Materials and Methods}

Experiment I (Seed germination). Fifty seeds, cv. Spring No. 3 of Earl's Favourite were placed on filter paper in a petri dish. The filter paper was moistened with 10 levels $(0,100,250,500,1,000,2,000,3,000,4,000$, 5,000 and $6,000 \mathrm{ppm} \mathrm{Cl}$ ) of sea water diluted with distilled water. Seeds were incubated at $25^{\circ} \mathrm{C}$. Treatments were replicated 5 times. The number of germinated seeds and the root elongation were recorded $: 2,3,4$ and 7 days after starting the test.

Experiment II (Seedlings). Fifteen germinated seeds, cv. Fall No.1 of Earl's Favourite were planted in a wooden box $(40 \times$ $40 \times 20 \mathrm{~cm}$ ) filled with sand on Sept. 3, 1975 . Seedlings were gradually thinned to 9 uniform plants per box by Sept. 13 . Six levels $(0,100,250,500,1,000$ and $2,000 \mathrm{ppm} \mathrm{Cl})$ of 
Table 1. Cation concentrations and EC in relation to sea water diluted with base nutrient solution.*

\begin{tabular}{|c|c|c|c|c|c|c|c|}
\hline \multicolumn{3}{|c|}{ Sea water concentrations } & \multicolumn{4}{|c|}{ Cations (ppm) } & \multirow{2}{*}{$\underset{(\mathrm{m} \mho / \mathrm{cm})}{\mathrm{EC}}$} \\
\hline $\begin{array}{c}\mathrm{Cl} \\
(\mathrm{ppm})\end{array}$ & $\begin{array}{l}\text { Osmotic potential } \\
\text { (bars) }\end{array}$ & $\%$ & $\mathrm{~K}$ & $\mathrm{Na}$ & $\mathrm{Ca}$ & $\mathrm{Mg}$ & \\
\hline 0 & -0.69 & 0 & 235 & 44 & 232 & 46 & 2.43 \\
\hline 50 & -0.76 & 0.25 & 236 & 69 & 233 & 49 & 2.44 \\
\hline 100 & -0.83 & 0.50 & 237 & 94 & 234 & 52 & 2. 61 \\
\hline 250 & -1.02 & 1.25 & 241 & 170 & 237 & 61 & 3.03 \\
\hline 500 & -1.35 & 2.50 & 246 & 296 & 242 & 78 & 3. 87 \\
\hline 1,000 & -2.02 & 5.00 & 257 & 548 & 252 & 109 & 4.96 \\
\hline 2,000 & -3.33 & 10.0 & 280 & 1,052 & 271 & 172 & 7.74 \\
\hline 3,000 & -4.66 & 15.0 & 302 & 1,556 & 291 & 235 & 10.75 \\
\hline 4,000 & -5.99 & 20.0 & 324 & 2,060 & 311 & 298 & 13. 20 \\
\hline 5,000 & -7.30 & 25.0 & 346 & 2,565 & 331 & 362 & 15.20 \\
\hline 6,000 & -8.63 & 30.0 & 369 & 3,069 & 350 & 425 & 17.45 \\
\hline \multicolumn{3}{|c|}{ Sea water $20,500 \mathrm{ppm} \mathrm{Cl}$} & 445 & 10,082 & 393 & 1,262 & 33.40 \\
\hline
\end{tabular}

*: Composition of base nutrient solution; $\mathrm{Na}_{2} \mathrm{HPO}_{4} \cdot 12 \mathrm{H}_{2} \mathrm{O}=0.5 \mathrm{mM}, \mathrm{K}_{2} \mathrm{SO}_{4}=3 \mathrm{mM}, \mathrm{Ca}\left(\mathrm{NO}_{3}\right)_{2} \cdot 4 \mathrm{H}_{2} \mathrm{O}=4 \mathrm{mM}$, $\mathrm{MgSO}_{4} \cdot 7 \mathrm{H}_{2} \mathrm{O}=2 \mathrm{mM}$, and minor elements ( $\mathrm{Mn}, \mathrm{Fe}, \mathrm{Zn}, \mathrm{Cu}, \mathrm{B}$ and $\mathrm{Mo}$ ). $\mathrm{pH}=6.0$

sea water diluted with base nutrient solution, as shown in Table 1, were applied to the sand medium from Sept. 4 to 19 , at which time the plant growth was measured and the sample taken. Treatments were replicated 5 times.

Experiment III (Sand culture). A uniform muskmelon seedling, cv. Spring No. 3 of Earl's Favourite grafted on Barnett Hill Favourite rootstock in the 4 leaf stage was transplanted to a wooden box $(40 \times 40 \times 20 \mathrm{~cm})$ filled with sand and placed in the greenhouse on May 29, 1975. Six levels (0, 50, 100, 250,500 and $1,000 \mathrm{ppm} \mathrm{Cl}$ ) of sea water diluted with base nutrient solution, as shown in Table 1, were applied to the sand medium once or twice a day according to the sand medium moisture from May 31 to Aug. 8, at which the muskmelons were harvested. Treatments were replicated 5 times. At the end of the experiment records were taken of salt injury symptoms of plants, top and root growth, fruit fresh weight and soluble solids. Major nutrient elements and osmotic potential of each plant part, and the chemical properties of the sand were determined as described previously (16).

Experiment IV (Nutrient solution culture). A uniform muskmelon seedling, cv. Spring No. 3 of Earl's Favourite grafted on Barnett Hill Favourite rootstock in the 4 leaf stage was transferred to each 13 liter pot, with 6 levels $(0,1,000,2,000,3,000,4,000$ and $5,000 \mathrm{ppm} \mathrm{Cl}$ ) of sea water diluted with base nutrient solution on Apr. 19, 1976. The experiment was continued in the greenhouse until July 8 . Treatments were replicated 6 times. The diluted sea water solution was replaced every one or two weeks and aerated continuously. At the end of the experiment, the same measurements as in Experiment III, plus transpiration rate and amount were measured as described previously (17).

\section{Results}

Seed germination (Table 2). The germination rate 2,3 and 4 days after starting the test was significantly higher at 0 to 3,000 $\mathrm{ppm} \mathrm{Cl}$ than at 4,000 to $6,000 \mathrm{ppm} \mathrm{Cl}$. After 7 days it was not significantly different from 0 to $5,000 \mathrm{ppm} \mathrm{Cl}$. Germination was most suppressed at $6,000 \mathrm{ppm} \mathrm{Cl}$, showing 22.0 , $76.0,84.0$ and $88.4 \%$ after $2,3,4$ and 7 days, respectively. Root elongation of germinated seeds was greatest at 100 and $250 \mathrm{ppm} \mathrm{Cl}$ followed by 0 and $500 \mathrm{ppm} \mathrm{Cl}$. Root elongation tended to become shorter with increasing sea water concentrations above $1,000 \mathrm{ppm} \mathrm{Cl}$.

Seedlings (Table 3). As shown in Table 3 , plant height of seedlings, number of leaves, and fresh weight of leaves, stem and whole seedlings were greatest at $0 \mathrm{ppm} \mathrm{Cl}$ and decreased as sea water concentrations 
Table 2. Effect of sea water concentrations on the germination rate of muskmelon seeds (\%).

\begin{tabular}{|c|c|c|c|c|c|}
\hline \multicolumn{2}{|c|}{ Sea water concentrations } & \multicolumn{4}{|c|}{ Days after starting the germination test } \\
\hline $\begin{array}{c}\mathrm{Cl} \\
(\mathrm{ppm})\end{array}$ & $\begin{array}{c}\text { Osmotic potential } \\
\text { (bars) }\end{array}$ & $2^{\mathrm{x}}$ & 3 & 4 & 7 \\
\hline 0 & 0 & $91.2^{\mathrm{a}}$ & $94.0^{\mathrm{abc}}$ & $94.8^{\mathrm{ab}}$ & $94.8^{\mathrm{a}}$ \\
\hline 100 & -0.14 & $87.6^{\mathrm{ab}}$ & $91.2^{\mathrm{bcd}}$ & $92.4^{\mathrm{abc}}$ & $92.4^{\mathrm{ab}}$ \\
\hline 250 & -0.33 & $93.2^{\mathbf{a}}$ & $96.4^{\mathrm{a}}$ & $96.4^{\mathrm{a}}$ & $96.4^{\mathrm{a}}$ \\
\hline 500 & -0.66 & 93. $6^{\mathrm{a}}$ & $96.0^{\mathrm{ab}}$ & $96.0^{\mathrm{a}}$ & $96.0^{\mathrm{a}}$ \\
\hline 1,000 & -1.33 & $92.0^{\mathrm{a}}$ & $95.2^{\mathrm{abc}}$ & $95.6^{\mathrm{ab}}$ & $95.6^{\mathbf{a}}$ \\
\hline 2,000 & -2.64 & $91.2^{\mathrm{a}}$ & $94.8^{\mathrm{abc}}$ & $95.6^{\mathrm{ab}}$ & $96.0^{\mathbf{a}}$ \\
\hline 3,000 & -3.97 & $88.0^{\mathrm{ab}}$ & $94.0^{\mathrm{abc}}$ & $95.2^{\mathrm{ab}}$ & $95.6^{\mathbf{a}}$ \\
\hline 4,000 & -5.30 & $81.6^{\mathrm{b}}$ & $90.8^{\mathrm{cd}}$ & $91.2^{\mathrm{bc}}$ & $92.4^{\mathrm{ab}}$ \\
\hline 5,000 & -6.61 & $55.2^{\mathrm{c}}$ & $88.4^{\mathrm{d}}$ & $89.6^{c}$ & $92.0^{\mathrm{ab}}$ \\
\hline 6,000 & -7.94 & $22.0^{\mathrm{d}}$ & $76.0^{\mathbf{e}}$ & $84.0^{\mathrm{d}}$ & $88.4^{\text {b }}$ \\
\hline
\end{tabular}

$\mathrm{x}$ : Mean separation in columns by Duncan's multiple range test, $5 \%$ level.

Table 3. Effect of sea water concentrations on the grow th of muskmelon seedlings in sand culture.

\begin{tabular}{|c|c|c|c|c|c|c|c|}
\hline \multicolumn{2}{|c|}{ Sea water concentrations } & \multirow{2}{*}{$\begin{array}{c}\text { Seedling } x \\
\text { height } \\
(\mathrm{cm})\end{array}$} & \multirow{2}{*}{$\begin{array}{l}\text { No. of } \\
\text { leaves }\end{array}$} & \multicolumn{4}{|c|}{ Fresh wt (g) } \\
\hline $\begin{array}{c}\mathrm{Cl} \\
(\mathrm{ppm})\end{array}$ & $\begin{array}{c}\text { Osmotic potential } \\
\text { (bars) }\end{array}$ & & & Leaves & Stem & Roots & $\begin{array}{l}\text { Whole } \\
\text { seedling }\end{array}$ \\
\hline 0 & -0.69 & $15.0^{\mathrm{a}}$ & 4. $1^{\mathrm{a}}$ & $4.7^{\mathrm{a}}$ & $4.7^{\mathrm{a}}$ & $0.32^{\mathrm{ab}}$ & $9.7^{\mathrm{a}}$ \\
\hline 100 & -0.83 & $13.3^{b}$ & $3.9^{\mathrm{ab}}$ & $4.2^{\mathrm{a}}$ & $4.2^{\mathrm{b}}$ & $0.36^{\mathrm{a}}$ & $8.8^{\mathrm{a}}$ \\
\hline 250 & -1.02 & $10.9^{c}$ & $3.8^{\mathrm{b}}$ & $3.7^{\mathrm{b}}$ & $3.5^{\mathrm{b}}$ & $0.32^{\mathrm{ab}}$ & $7.5^{\mathrm{b}}$ \\
\hline 500 & -1.35 & $9.3^{\mathrm{d}}$ & $3.2^{\mathrm{c}}$ & $2.9^{\mathrm{c}}$ & $2.7^{\mathrm{c}}$ & $0.24^{\mathrm{bc}}$ & $5.8^{\mathrm{c}}$ \\
\hline 1,000 & -2.02 & $6.7^{\mathrm{e}}$ & $2.3^{\mathrm{d}}$ & $1.5^{\mathrm{d}}$ & 1. $2^{\mathrm{d}}$ & $0.18^{\mathrm{cd}}$ & $2.9^{d}$ \\
\hline 2,000 & -3.33 & $5.5^{\mathrm{e}}$ & $2.0^{\mathrm{d}}$ & $0.9^{\mathrm{d}}$ & $0.7^{\mathrm{e}}$ & $0.12^{\mathrm{d}}$ & $1.7^{\mathrm{d}}$ \\
\hline
\end{tabular}

$\mathrm{x}$ : The same as Table 2 .

Table 4. Effect of sea water concentrations on the grow th of muskmelons in sand culture.

\begin{tabular}{|c|c|c|c|c|c|c|c|c|c|c|}
\hline \multicolumn{2}{|c|}{$\begin{array}{c}\text { Sea water } \\
\text { concentrations }\end{array}$} & \multicolumn{2}{|c|}{ Plant height ${ }^{\mathrm{x}}(\mathrm{cm})$} & \multicolumn{5}{|c|}{ Fresh wt (g) } & \multirow{2}{*}{$\begin{array}{c}\text { Soluble } \\
\text { solids } \\
\text { of fruit } \\
(\%)\end{array}$} & \multirow{2}{*}{$\begin{array}{c}\text { Salt }^{\mathrm{Y}} \\
\text { in jury } \\
\text { symptoms }\end{array}$} \\
\hline $\begin{array}{c}\mathrm{Cl} \\
(\mathrm{ppm})\end{array}$ & $\begin{array}{l}\text { Osmotic } \\
\text { potential } \\
\text { (bars) }\end{array}$ & June 17 & Aug. 8 & Leaves & Stem & Roots & Fruit & $\begin{array}{l}\text { whole } \\
\text { plant }\end{array}$ & & \\
\hline 0 & -0.69 & $136.2^{\mathrm{a}}$ & $143.2^{\mathrm{a}}$ & $503^{a b}$ & $388^{\mathrm{ab}}$ & $30^{\mathrm{ab}}$ & $1,660^{\mathrm{a}}$ & $2,581^{\mathrm{ab}}$ & $13.6^{\mathrm{b}}$ & 0 \\
\hline 100 & -0.83 & $134.6^{\mathrm{a}}$ & $143.0^{\mathrm{a}}$ & $552^{a}$ & $431^{\mathrm{a}}$ & $32^{\mathbf{a}}$ & $1,687^{\mathrm{a}}$ & $2,702^{\mathbf{a}}$ & $14.4^{\mathrm{b}}$ & 0 \\
\hline 250 & -1.02 & $128.4^{\mathrm{b}}$ & $142.8^{\mathrm{a}}$ & $494^{\mathrm{ab}}$ & $406^{\mathrm{ab}}$ & $28^{a b}$ & $1,528^{\mathrm{ab}}$ & $2,456^{\mathbf{b}}$ & $14.9^{\mathrm{b}}$ & 0 \\
\hline 500 & -1.35 & $128.4^{\mathrm{b}}$ & 137. $2^{\mathbf{a b}}$ & $488^{\mathrm{ab}}$ & $374^{\mathrm{bc}}$ & $25^{\mathrm{abc}}$ & $1,336^{\mathrm{bc}}$ & $2,223^{\mathrm{c}}$ & $15.8^{\mathrm{a}}$ & $0 \sim 0.5$ \\
\hline 1,000 & -2.02 & $125.6^{\mathrm{b}}$ & $131.4^{\mathrm{b}}$ & $431^{\mathrm{b}}$ & $335^{\mathrm{c}}$ & $21^{b c}$ & $1,147^{\mathrm{cd}}$ & $1,934^{\mathrm{d}}$ & $15.7^{\mathrm{a}}$ & $1 \sim 2$ \\
\hline 2,000 & -3.33 & 109. $0^{c}$ & $123.0^{c}$ & $344^{\mathrm{c}}$ & $282^{\mathrm{d}}$ & $15^{\mathrm{c}}$ & $969^{d}$ & $1,610^{\mathrm{e}}$ & $15.7^{\mathrm{a}}$ & $2 \sim 3$ \\
\hline
\end{tabular}

$\mathrm{x}$ : The same as Table 2 .

$\mathrm{y}: 0=$ None, $3=$ Very severe.

increased.

\section{Sand culture}

Growth (Table 4). Whole plant and fruit fresh weight were greatest at 0 and $50 \mathrm{ppm}$ $\mathrm{Cl}$ and decreased with increasing sea water concentrations from 100 to $1,000 \mathrm{ppm} \mathrm{Cl}$. Plant height, and fresh weight of leaves, stem and roots were similar to the whole plant fresh weight. Soluble solids of fruit were higher at 250 to $1,000 \mathrm{ppm} \mathrm{Cl}$ than at 0 to $100 \mathrm{ppm} \mathrm{Cl}$. The salt injury, such as wilting of leaf margins, and chlorosis and necrosis on the leaves, was observed at 250 to 1,000 ppm $\mathrm{Cl}$ and became more severe with increasing sea water concentrations.

Osmotic potential and $\mathrm{Cl}$ content of each plant part (Table 5). Osmotic potential of leaves and roots decreased with increasing sea water concentrations. Fruit osmotic potential was not affected by treatments. Root osmotic potential was higher than that of leaves. $\mathrm{Cl}$ content in the plant parts increased as sea water concentrations increased. The $\mathrm{Cl}$ was highest in roots followed by leaves and 
Table 5. Effect of sea water concentrations on the osmotic potential and $\mathrm{Cl}$ content of each plant part of muskmelons in sand culture.

\begin{tabular}{r|c|c|c|c|c|c|c}
\hline \hline \multicolumn{2}{c|}{ Sea water concentrations } & \multicolumn{2}{c|}{ Osmotic potential (bars) } & \multicolumn{3}{c}{ Cl content (\% of dry matter) } \\
\cline { 2 - 8 } $\begin{array}{c}\text { Cl } \\
(\mathrm{ppm})\end{array}$ & $\begin{array}{c}\text { Osmotic potential } \\
\text { (bars) }\end{array}$ & Leaves & Roots & Fruit & Leaves & Roots & Fruit \\
\hline 0 & -0.69 & $-11.2^{\mathrm{a}}$ & -4.8 & $-15.0^{\mathrm{a}}$ & $0.41^{\mathrm{d}}$ & $1.75^{\mathrm{d}}$ & $0.71^{\mathrm{d}}$ \\
50 & -0.76 & $-11.8^{\mathrm{a}}$ & -5.5 & $-15.5^{\mathrm{ab}}$ & $0.67^{\mathrm{d}}$ & $2.13^{\mathrm{cd}}$ & $0.73^{\mathrm{cd}}$ \\
100 & -0.83 & $-12.0^{\mathrm{ab}}$ & -5.7 & $-14.9^{\mathrm{a}}$ & $0.80^{\mathrm{d}}$ & $2.31^{\mathrm{c}}$ & $0.76^{\mathrm{cd}}$ \\
250 & -1.02 & $-13.0^{\mathrm{b}}$ & -6.7 & $-15.8^{\mathrm{ab}}$ & $1.53^{\mathrm{c}}$ & $3.54^{\mathrm{b}}$ & $1.02^{\mathrm{bc}}$ \\
500 & -1.35 & $-14.5^{\mathrm{c}}$ & -11.3 & $-16.2^{\mathrm{ab}}$ & $2.19^{\mathrm{b}}$ & $4.98^{\mathrm{a}}$ & $1.16^{\mathrm{ab}}$ \\
1,000 & -2.02 & $-15.0^{\mathrm{c}}$ & -11.3 & $-16.5^{\mathrm{b}}$ & $3.48^{\mathrm{a}}$ & $5.45^{\mathrm{a}}$ & $1.41^{\mathrm{a}}$ \\
\hline
\end{tabular}

$\mathrm{x}:$ The same as Table 2 .

$\mathrm{y}$ : Not subjected to statistical analysis due to composite sampling from each treatment.

Table 6. Effect of sea water concentrations on the major nutrient elements of muskmelon leaves in sand culture ( $\%$ of dry matter).

\begin{tabular}{r|c|c|c|c|c|c|c}
\hline \hline \multicolumn{2}{c|}{ Sea water concentrations } & \multirow{2}{*}{ Total-N } & P & K & Na & Ca & $\mathrm{Mg}$ \\
\cline { 1 - 5 } $\begin{array}{c}\text { Cl } \\
(\mathrm{ppm})\end{array}$ & $\begin{array}{c}\text { Osmotic potential } \\
\text { (bars) }\end{array}$ & & & & & \\
\hline 0 & -0.69 & $3.39^{\mathrm{ab}}$ & $0.43^{\mathrm{b}}$ & $1.35^{\mathrm{a}}$ & $0.25^{\mathrm{c}}$ & $6.99^{\mathrm{a}}$ & $2.24^{\mathrm{b}}$ \\
50 & -0.83 & $3.74^{\mathrm{a}}$ & $0.44^{\mathrm{b}}$ & $1.41^{\mathrm{a}}$ & $0.27^{\mathrm{c}}$ & $6.93^{\mathrm{a}}$ & $2.21^{\mathrm{b}}$ \\
100 & -1.02 & $3.41^{\mathrm{ab}}$ & $0.45^{\mathrm{b}}$ & $1.31^{\mathrm{a}}$ & $0.32^{\mathrm{c}}$ & $6.43^{\mathrm{a}}$ & $2.22^{\mathrm{b}}$ \\
250 & -1.35 & $3.56^{\mathrm{ab}}$ & $0.48^{\mathrm{ab}}$ & $1.29^{\mathrm{a}}$ & $0.41^{\mathrm{c}}$ & $6.33^{\mathrm{a}}$ & $2.25^{\mathrm{b}}$ \\
500 & -2.02 & $3.30^{\mathrm{bc}}$ & $0.52^{\mathrm{ab}}$ & $1.37^{\mathrm{a}}$ & $0.66^{\mathrm{b}}$ & $6.47^{\mathrm{a}}$ & $2.40^{\mathrm{a}}$ \\
1,000 & -3.33 & $2.94^{\mathrm{c}}$ & $0.59^{\mathrm{a}}$ & $1.37^{\mathrm{a}}$ & $0.99^{\mathrm{a}}$ & $6.30^{\mathrm{a}}$ & $2.39^{\mathrm{a}}$ \\
\hline
\end{tabular}

$\mathrm{x}$ : The same as Table 2 .

Table 7. Chemical properties of the sand at the end of the experiment (air dried soil basis).

\begin{tabular}{|c|c|c|c|c|c|c|c|c|c|c|}
\hline \multicolumn{2}{|c|}{ Sea water concentrations } & \multirow{2}{*}{$\begin{array}{l}\mathrm{NO}_{3}-\mathrm{N}^{\mathrm{X}} \\
(\mathrm{ppm})\end{array}$} & \multirow{2}{*}{$\begin{array}{c}\mathrm{P}(\text { Truog) } \\
(\mathrm{ppm})\end{array}$} & \multicolumn{4}{|c|}{ Exchangeable cations $(\mathrm{me} / 100 \mathrm{~g})$} & \multirow{2}{*}{$\begin{array}{c}\mathrm{Cl} \\
(\mathrm{ppm})\end{array}$} & \multirow{2}{*}{$\begin{array}{l}\operatorname{EC}(1: 5) \\
(\mathrm{m} \mho / \mathrm{cm})\end{array}$} & \multirow{2}{*}{$\begin{array}{c}\mathrm{pH} \\
\left(\mathrm{H}_{2} \mathrm{O}\right)\end{array}$} \\
\hline $\begin{array}{c}\mathrm{Cl} \\
(\mathrm{ppm})\end{array}$ & $\begin{array}{c}\text { Osmotic } \\
\text { potential (bars) } \\
\end{array}$ & & & $\mathrm{K}$ & $\mathrm{Na}$ & $\mathrm{Ca}$ & $\mathrm{Mg}$ & & & \\
\hline 0 & -0.69 & $43^{\mathrm{ab}}$ & $32^{\mathrm{ab}}$ & $0.26^{\mathrm{bc}}$ & $0.24^{d}$ & $2.08^{\mathrm{a}}$ & $0.47^{\mathrm{b}}$ & $50^{d}$ & $0.46^{\mathrm{a}}$ & $5.9^{a}$ \\
\hline 50 & -0.83 & $43^{\mathrm{ab}}$ & $26^{\mathrm{b}}$ & $0.24^{\mathrm{c}}$ & $0.26^{\mathrm{d}}$ & $1.75^{\mathrm{b}}$ & $0.45^{\mathrm{b}}$ & $66^{\mathrm{d}}$ & $0.44^{\mathrm{a}}$ & $5.6^{\mathrm{a}}$ \\
\hline 100 & -1.02 & $53^{\mathrm{a}}$ & $28^{\mathrm{b}}$ & $0.29^{\mathrm{ab}}$ & $0.33^{d}$ & $1.83^{\mathrm{b}}$ & $0.46^{\mathrm{b}}$ & $70^{\mathrm{d}}$ & $0.44^{\mathrm{a}}$ & $5.9^{\mathrm{a}}$ \\
\hline 250 & -1.35 & $51^{\mathrm{a}}$ & $26^{\mathrm{b}}$ & $0.30^{\mathrm{a}}$ & $0.51^{\mathrm{c}}$ & 1. $44^{\mathrm{c}}$ & $0.47^{\mathrm{b}}$ & $201^{c}$ & $0.46^{\mathrm{a}}$ & $6.1^{\mathrm{a}}$ \\
\hline 500 & -2.02 & $47^{\mathrm{ab}}$ & $29^{b}$ & $0.28^{\mathrm{ab}}$ & $0.78^{b}$ & $1.08^{\mathrm{d}}$ & $0.50^{\mathrm{ab}}$ & $320^{b}$ & $0.44^{\mathrm{a}}$ & $6.3^{\mathrm{a}}$ \\
\hline 1,000 & -3.33 & $38^{\mathrm{b}}$ & $36^{\mathrm{a}}$ & $0.27^{\mathrm{abc}}$ & $1.11^{\mathrm{a}}$ & $0.80^{\mathrm{e}}$ & $0.56^{\mathrm{a}}$ & $487^{\mathrm{a}}$ & $0.46^{\mathrm{a}}$ & $6.0^{\mathrm{a}}$ \\
\hline
\end{tabular}

$\mathrm{x}$ : The same as Table 2 .

then fruit. $\mathrm{Cl}$ content at $0 \mathrm{ppm} \mathrm{Cl}$ was 0.41 , 1.75 and $0.71 \%$ in leaves, roots and fruit, respectively. This $\mathrm{Cl}$ seemed to result from the reagents and tap water used for making nutrient solution, and from the sand used as a medium.

Major nutrient elements in leaves (Table 6). Content of $\mathrm{Na}$ and $\mathrm{P}$ increased with increasing sea water concentrations. Total$\mathrm{N}$ content was lower at $1,000 \mathrm{ppm} \mathrm{Cl}$, and $\mathrm{Mg}$ content was higher at 500 and $1,000 \mathrm{ppm}$ Cl. There was no significant difference in $\mathrm{K}$ and $\mathrm{Ca}$ content.

Chemical properties of sand at the end of the experiment (Table 7). Amounts of $\mathrm{Cl}$ and exchangeable $\mathrm{Na}$ and $\mathrm{Mg}$ increased, and exchangeable $\mathrm{Ca}$ content decreased with increasing sea water concentrations. There was no significant difference in $\mathrm{EC}, \mathrm{pH}$, $\mathrm{NO}_{3}-\mathrm{N}, \mathrm{P}$ and $\mathrm{K}$ levels.

\section{Nutrient solution culture}

Growth (Table 8). The salt injury, such as cupping of leaf margins and necrosis of leaves, was observed above $3,000 \mathrm{ppm} \mathrm{Cl}$ and the degree of injury became more severe with increasing sea water concentrations. Plant height, fresh weight of leaves, stem, roots and fruit, and whole plant dry weight at harvest decreased as sea water concentrations increased. The growth at $5,000 \mathrm{ppm} \mathrm{Cl}$ was 
Table 8. Effect of sea water concentrations on the grow th of muskmelons in nutrient solution culture.

\begin{tabular}{|c|c|c|c|c|c|c|c|c|c|c|c|}
\hline \multicolumn{2}{|c|}{$\begin{array}{c}\text { Sea water } \\
\text { concentrations }\end{array}$} & \multirow{2}{*}{$\begin{array}{l}\text { Plant } \mathrm{x} \\
\text { height at } \\
\text { harvest } \\
(\mathrm{cm})\end{array}$} & \multirow{2}{*}{$\begin{array}{l}\text { Leaf } \\
\text { fresh } \\
\text { wt }(g)\end{array}$} & \multirow{2}{*}{$\begin{array}{l}\text { Root } \\
\text { fresh } \\
\operatorname{wt}(g)\end{array}$} & \multirow{2}{*}{$\begin{array}{l}\text { Whole } \\
\text { plant } \\
\text { dry } \\
\text { wt }(g)\end{array}$} & \multirow{2}{*}{$\begin{array}{l}\text { Dry matter } \\
\text { content of } \\
\text { leaves } \\
(\%)\end{array}$} & \multirow{2}{*}{$\begin{array}{c}\text { Salt }^{\mathrm{Y}} \\
\text { in jury } \\
\text { symptoms }\end{array}$} & \multicolumn{4}{|c|}{ Fruit } \\
\hline $\begin{array}{c}\mathrm{Cl} \\
(\mathrm{ppm})\end{array}$ & $\begin{array}{l}\text { Osmotic } \\
\text { potential } \\
\text { (bars) }\end{array}$ & & & & & & & $\begin{array}{l}\text { Fresh } \\
\text { wt (g) }\end{array}$ & $\begin{array}{c}\text { Soluble } \\
\text { solids } \\
(\%)\end{array}$ & $\begin{array}{l}\text { External }{ }^{\mathrm{W}} \\
\text { appearance }\end{array}$ & $\begin{array}{l}\text { Salty }{ }^{2} \\
\text { taste }\end{array}$ \\
\hline 0 & -0.69 & $109^{a}$ & $503^{a}$ & $238^{a}$ & $255^{\mathrm{a}}$ & $14.6^{\mathrm{a}}$ & 0 & $1,518^{\mathrm{a}}$ & $13.9^{b}$ & $7.2^{\mathrm{a}}$ & 0 \\
\hline 1,000 & -2.20 & $101^{b}$ & $398^{b}$ & $137^{\mathrm{b}}$ & $225^{\mathbf{b}}$ & $14.6^{\mathrm{a}}$ & 0 & $1,484^{\mathrm{a}}$ & $14.1^{b}$ & $7.8^{\mathrm{a}}$ & $0 \sim 0.5$ \\
\hline 2,000 & -3.33 & $84^{\mathrm{c}}$ & $376^{\mathrm{b}}$ & $131^{b}$ & $193^{c}$ & $14.5^{\mathrm{a}}$ & 0 & $1,299^{b}$ & $14.4^{\mathrm{ab}}$ & $5.6^{\mathrm{b}}$ & $1 \sim 2$ \\
\hline 3,000 & -4.66 & $74^{\mathrm{d}}$ & $256^{\mathrm{c}}$ & $66^{\mathrm{c}}$ & $122^{\mathrm{d}}$ & $14.4^{\mathrm{a}}$ & 0.5 & $922^{c}$ & $14.8^{\mathrm{ab}}$ & $3.8^{\mathrm{c}}$ & $2 \sim 3$ \\
\hline 4,000 & -5.99 & $68^{\mathrm{de}}$ & $216^{\mathrm{c}}$ & $49^{c}$ & $104^{\mathrm{d}}$ & $14.3^{\mathrm{a}}$ & $1 \sim 2$ & $726^{\mathrm{d}}$ & $15.2^{\mathrm{a}}$ & $1.7^{\mathrm{d}}$ & 4 \\
\hline 5,000 & -7.30 & $66^{\mathbf{e}}$ & $204^{c}$ & $59^{c}$ & $34^{\mathbf{e}}$ & $10.0^{\mathrm{b}}$ & 3 & $*$ & $*$ & * & $*$ \\
\hline
\end{tabular}

$\mathrm{x}$ : The same as Table 2. $\mathrm{y}: 0=$ None, $3=$ Very severe. $\mathrm{z}: 0=$ None, $4=$ Very salty. $\mathrm{w}:$ Full score $=10$.

$*:$ Fruit did not set due to poor growth.

Table 9. Effect of sea water concentrations on the osmotic potential of leaves and roots, and transpiration of muskmelons in nutrient solution culture.

\begin{tabular}{|c|c|c|c|c|c|c|c|}
\hline \multicolumn{2}{|c|}{ Sea water concentrations } & \multicolumn{2}{|c|}{$\begin{array}{c}\text { Osmotic potentialx } \\
\text { (bars) }\end{array}$} & \multicolumn{3}{|c|}{$\begin{array}{l}\text { Transpiration amount } \\
\text { (g. water lost/plant) }\end{array}$} & \multirow{2}{*}{$\begin{array}{l}\text { Transpiration rate } \\
\begin{array}{c}\text { g. water lost } / \\
\text { g. leaf dry wt) }\end{array} \\
\text { July } 1^{z}\end{array}$} \\
\hline $\begin{array}{c}\mathrm{Cl} \\
(\mathrm{ppm})\end{array}$ & $\begin{array}{c}\text { Osmotic potential } \\
\text { (bars) }\end{array}$ & Leaves & Roots & May $28^{\mathrm{W}}$ & May $29^{\mathrm{x}}$ & June $5^{y}$ & \\
\hline 0 & -0.69 & $-8.6^{\mathrm{e}}$ & $-4.2^{b}$ & $1,834^{\mathrm{a}}$ & $1,156^{\mathrm{a}}$ & $225^{\mathrm{a}}$ & $15.6^{\mathrm{a}}$ \\
\hline 1,000 & -2.02 & $-9.2^{\mathrm{d} e}$ & $-4.0^{\mathrm{b}}$ & $1,408^{b}$ & $982^{\mathrm{b}}$ & $158^{\mathrm{b}}$ & $14.8^{\mathrm{ab}}$ \\
\hline 2,000 & -3.33 & $-11.3^{\mathrm{cd}}$ & $-4.0^{\mathrm{b}}$ & $1,211^{\mathrm{c}}$ & $816^{\mathrm{c}}$ & $158^{\mathrm{b}}$ & $13.2^{\mathrm{cd}}$ \\
\hline 3,000 & -4.66 & $-12.5^{c}$ & $-4.5^{\mathrm{b}}$ & $745^{d}$ & $535^{d}$ & $100^{\mathrm{c}}$ & $13.7^{\mathrm{bc}}$ \\
\hline 4,000 & -5.99 & $-15.0^{\mathrm{b}}$ & $-4.8^{\mathrm{b}}$ & $560^{\mathrm{e}}$ & $422^{\mathrm{e}}$ & $98^{\mathrm{c}}$ & $12.0^{\mathrm{d}}$ \\
\hline 5,000 & -7.30 & $-24.2^{\mathrm{a}}$ & $-6.2^{\mathrm{a}}$ & $186^{\mathrm{f}}$ & $128^{\mathrm{f}}$ & $57^{\mathrm{d}}$ & $3.9^{\mathrm{e}}$ \\
\hline
\end{tabular}

$\mathrm{x}$ : The same as Table 2. $\mathrm{w}$ : Clear during the day. $\mathrm{x}$ : Clear in the morning, then cloudy in the afternoon. $\mathrm{y}$ : Rainy during the day. $\mathrm{z}$ : Clear during the day.

Table 10. Effect of sea water concentrations on the $\mathrm{Na}$ and $\mathrm{Cl}$ content in different parts of muskmelons in nutrient solution culture ( $\%$ of dry matter).

\begin{tabular}{|c|c|c|c|c|c|c|c|c|c|}
\hline \multicolumn{2}{|c|}{$\begin{array}{c}\text { Sea water } \\
\text { concentrations }\end{array}$} & \multicolumn{4}{|c|}{$\mathrm{Na}$} & \multicolumn{4}{|c|}{$\mathrm{Cl}$} \\
\hline $\begin{array}{c}\mathrm{Cl} \\
(\mathrm{ppm})\end{array}$ & $\begin{array}{l}\text { Osmotic } \\
\text { potential } \\
\text { (bars) }\end{array}$ & Leaves $^{\mathrm{X}}$ & Stem & Roots & Fruit & Leaves & Stem & Roots & Fruit \\
\hline 0 & -0.69 & $0.16^{\mathrm{c}}$ & $1.17^{\mathrm{e}}$ & $0.56^{\mathrm{d}}$ & $0.44^{\mathrm{d}}$ & $0.33^{e}$ & $3.01^{\mathrm{e}}$ & $0.43^{\mathrm{c}}$ & $0.54^{\mathrm{d}}$ \\
\hline 1,000 & -2.02 & $0.44^{\mathrm{bc}}$ & 3. $88^{\mathrm{d}}$ & 1. $28^{\mathrm{c}}$ & $0.94^{\mathrm{c}}$ & 1. $11^{\mathrm{de}}$ & $8.96^{\mathrm{d}}$ & $0.99^{\mathrm{c}}$ & $1.07^{\mathrm{d}}$ \\
\hline 2,000 & -3.33 & $0.56^{\mathrm{bc}}$ & 6. $E 0^{\mathrm{c}}$ & $1.81^{\mathrm{b}}$ & $1.65^{\mathrm{b}}$ & 2. $22^{\mathrm{cd}}$ & $12.63^{\mathrm{c}}$ & $1.90^{\mathrm{b}}$ & $2.15^{\mathrm{c}}$ \\
\hline 3,000 & -4.66 & $0.66^{\mathrm{b}}$ & $7.40^{\mathrm{c}}$ & $1.86^{\mathrm{b}}$ & $1.74^{\mathrm{b}}$ & $2.95^{\mathrm{c}}$ & $14.52^{\mathrm{b}}$ & $2.04^{\mathrm{b}}$ & $2.90^{\mathrm{b}}$ \\
\hline 4,000 & -5.99 & $0.81^{b}$ & 9. $80^{\mathrm{b}}$ & $2.04^{\mathrm{b}}$ & 2. $16^{\mathrm{a}}$ & 4. $62^{b}$ & $19.17^{\mathrm{a}}$ & 2. $64^{\mathrm{a}}$ & $3.65^{\mathrm{a}}$ \\
\hline 5,000 & -7.30 & $4.96^{\mathrm{a}}$ & $10.93^{\mathrm{a}}$ & $2.35^{\mathrm{a}}$ & $*$ & $15.95^{\mathrm{a}}$ & $19.53^{\mathrm{a}}$ & 2. $85^{\mathrm{a}}$ & * \\
\hline
\end{tabular}

$\mathrm{x}$ : The same as Table 2 .

* : Fruit did not set due to poor growth.

most suppressed and the whole plant dry weight was $34 \mathrm{~g}$ which is $13 \%$ of that at $0 \mathrm{ppm} \mathrm{Cl}$. The fruit at $5,000 \mathrm{ppm} \mathrm{Cl}$ did not set due to poor growth. External appearance of fruit became poorer and the fruit soluble solids increased as sea water concentrations increased. Panel tests showed that the taste became saltier with increasing sea water concentrations. All panelists reported severely salty taste at $4,000 \mathrm{ppm} \mathrm{Cl}$.

Osmotic potential of leaves and roots, and transpiration rate (Table 9). Osmotic potential of leaves decreased with increasing sea water concentrations. It was especially low at $5,000 \mathrm{ppm} \mathrm{Cl}$ showing -24.1 bars. However, that of roots was not significantly different from 0 to $4,000 \mathrm{ppm} \mathrm{Cl}$ and decreased at only $5,000 \mathrm{ppm} \mathrm{Cl}$ showing -6.2 bars. Root osmotic potential was higher than that of leaves. The transpiration amount (g. water lost/plant) measured on May 28, a clear day, decreased markedly with increasing sea water 
Table 11. Effect of sea water concentrations on the major nutrient elements of muskmelon leaves in nutrient solution culture ( $\%$ of dry matter).

\begin{tabular}{c|c|c|c|c|c|c}
\hline \hline \multicolumn{2}{c|}{ Sea water concentrations } & Total-N & $\mathrm{P}$ & $\mathrm{K}$ & $\mathrm{Ca}$ & $\mathrm{Mg}$ \\
\hline $\begin{array}{c}\mathrm{Cl} \\
(\mathrm{ppm})\end{array}$ & $\begin{array}{c}\text { Osmotic potential } \\
\text { (bars) }\end{array}$ & Ta & & & \\
\hline 0 & -0.69 & $2.87^{\mathrm{a}}$ & $1.24^{\mathrm{a}}$ & $1.87^{\mathrm{b}}$ & $11.38^{\mathrm{a}}$ & $1.71^{\mathrm{c}}$ \\
1,000 & -2.02 & $2.57^{\mathrm{bc}}$ & $1.36^{\mathrm{a}}$ & $1.26^{\mathrm{c}}$ & $8.52^{\mathrm{b}}$ & $2.26^{\mathrm{c}}$ \\
2,000 & -3.33 & $2.74^{\mathrm{ab}}$ & $1.16^{\mathrm{a}}$ & $0.63^{\mathrm{d}}$ & $8.95^{\mathrm{b}}$ & $3.01^{\mathrm{b}}$ \\
3,000 & -4.66 & $2.52^{\mathrm{c}}$ & $1.12^{\mathrm{a}}$ & $0.55^{\mathrm{d}}$ & $9.14^{\mathrm{b}}$ & $3.33^{\mathrm{a}}$ \\
4,000 & -5.99 & $2.49^{\mathrm{c}}$ & $0.94^{\mathrm{b}}$ & $0.50^{\mathrm{d}}$ & $9.33^{\mathrm{b}}$ & $3.35^{\mathrm{a}}$ \\
5,000 & -7.30 & $2.14^{\mathrm{d}}$ & $1.29^{\mathrm{a}}$ & $3.13^{\mathrm{a}}$ & $4.49^{\mathrm{c}}$ & $2.98^{\mathrm{b}}$ \\
\hline
\end{tabular}

$\mathrm{x}$ : The same as Table 2.

concentrations. The transpiration rate (g. water lost/g. leaf dry weight) measured on July 1 did not decrease so much as the transpiration amount did.

Content of $\mathrm{Na}$ and $\mathrm{Cl}$ in each plant part (Table 10$). \mathrm{Na}$ and $\mathrm{Cl}$ content in leaves, stem, roots and fruit increased as sea water concentrations increased. $\mathrm{Na}$ and $\mathrm{Cl}$ in leaves were especially higher at $5,000 \mathrm{ppm} \mathrm{Cl}$ than at $4,000 \mathrm{ppm} \mathrm{Cl}$. $\mathrm{Na}$ and $\mathrm{Cl}$ accumulated most in stem, less in roots and fruit, and least in leaves. $\mathrm{Cl}$ content in leaves was much higher than $\mathrm{Na}$. The $\mathrm{Na}$ and $\mathrm{Cl}$ content in leaves at $4,000 \mathrm{ppm} \mathrm{Cl}$ was 5.0 and 14.0 times, as compared to $0 \mathrm{ppm} \mathrm{Cl}$, respectively.

Major nutrient elements in leaves (Table 11). Total-N, $\mathrm{K}$ and $\mathrm{Ca}$ content in leaves tended to decrease and $\mathrm{Mg}$ content increased, as sea water concentrations increased from 0 to $4,000 \mathrm{ppm} \mathrm{Cl}$. The content of $\mathrm{K}$ was high and $\mathrm{Ca}$ was low at $5,000 \mathrm{ppm} \mathrm{Cl}$.

\section{Discussion}

Diluted sea water, up to $3,000 \mathrm{ppm} \mathrm{Cl}$, did not delay initial emergence and did not decrease the ultimate germination rate below $5,000 \mathrm{ppm} \mathrm{Cl}$. It seemed that there is no general relation between the salt tolerance of a plant during the later stage of growth and that of seeds during germination $(1,5)$. Muskmelon seeds were more tolerant of salinity than tomato seeds (17) and less tolerant than green soybean seeds (16). The salt tolerance of plants during germination might be related to the size of seeds from these experiments.

It was found that lower concentrations, 250 or $500 \mathrm{ppm} \mathrm{Cl}$, of diluted sea water stimulated the seedling growth of green soy- beans (16) and vegetative growth during the early growth stage of tomatoes (17). Similar results were obtained by other investigators $(8,21,25)$. However, muskmelon seedlings were largest at $0 \mathrm{ppm} \mathrm{Cl}$ when expressed by plant height or fresh weight of whole seedlings in the present experiment. Also in pre-nutrient solution culture (12), the vegetative growth was not stimulated by lower concentrations of diluted sea water. These results may indicate that the response of muskmelons to diluted sea water is somewhat different from tomatoes and green soybeans.

Soluble solids of fruit tended to be higher with increasing sea water concentrations in sand and nutrient solution cultures. This result may be related to the fruit size and weight. This reason is that small fruit must concentrate the soluble solids, and salinity did not seem to affect the content of sucrose, glucose and fructose (19).

Salt injury was observed above $250 \mathrm{ppm}$ $\mathrm{Cl}$ in sand culture, and above $3,000 \mathrm{ppm} \mathrm{Cl}$ in nutrient solution culture. In these cases, leaf $\mathrm{Na}$ and $\mathrm{Cl}$ content was 0.41 and $1.53 \%$ at $250 \mathrm{ppm} \mathrm{Cl}$ in sand culture, and 0.66 and $2.95 \%$ at $3,000 \mathrm{ppm} \mathrm{Cl}$ in nutrient solution culture, respectively. In a soil culture experiment (18), the minimum $\mathrm{Na}$ and $\mathrm{Cl}$ content in leaves where the salt injury was observed was about 0.5 and $3.0 \%$, respectively. In both soil and nutrient solution cultures, the injury seemed to be correlated with leaf $\mathrm{Na}$ and/or $\mathrm{Cl}$ content and to be induced by more than $0.5 \% \mathrm{Na}$ and/or $3.0 \%$ $\mathrm{Cl}$. However, the result in sand culture was not identical with soil and nutrient solution cultures. The relationships be- 
tween the injury and leaf $\mathrm{Na}$ and/or $\mathrm{Cl}$ content were disputable in these experiments, although leaf $\mathrm{Na}$ and/or $\mathrm{Cl}$ content may affect the degree of injury. Much more $\mathrm{Na}$ and $\mathrm{Cl}$ would have been accumulated in injured leaves because analyses were made of pooled samples. Ehlig and Bernstein (6) reported that high concentrations of $\mathrm{Cl}$ in the substrate caused the marginal burn on mature strawberry leaves and $\mathrm{Na}$ also produced some marginal burn. However, Bernstein et al. stated that there was no relationships between $\mathrm{Cl}$ content and salt tolerance in green beans (3), and that growth responses attributed to salinity were due primarily to physiological scarcity of water rather than to any derangement in mineral nutrition, although the $\mathrm{Cl}$ content showed the best relationship to the reduction in size of the lettuce plant (2). Shimada (23) also suggested that the reduction of cucumber fresh weight was due to a functional disorder of water absorption mechanisms in roots by high salinity and that visible symptoms of leaves were due to accumulation of excess salts.

Dry matter content of leaves in nutrient solution culture was not significantly different from 0 to $4,000 \mathrm{ppm} \mathrm{Cl}$, and only at 5,000 $\mathrm{ppm} \mathrm{Cl}$ it decreased. This reduction may be caused by the lack of fruit set due to poor growth. Decreased top dry matter content in high salt treatments with some fruiting vegetables was reported by Osawa (20). Nieman (15) observed that $\mathrm{NaCl}$ increased the succulence of some crop plants and stated that the increase is a fairly common plant response to high levels of chloride salts. The increase of tomato leaf water content suggests an increase in cell size (8).

Osmotic potential of leaves in nutrient solution culture was gradually decreased with increasing sea water concentrations, and dry matter content, except for $5,000 \mathrm{ppm} \mathrm{Cl}$, was not significantly different. At $5,000 \mathrm{ppm} \mathrm{Cl}$ the osmotic potential of leaves was markedly decreased in spite of decreased dry matter content. These results suggest that low osmotic potentials of leaves resulted from an increase in ion concentrations. Ion uptake is apparently the predominant factor in the osmotic adjustment of the plant sap (4).
Osmotic potentials of roots were higher than those of leaves in sand and nutrient solution cultures, and were not significantly different from 0 to $4,000 \mathrm{ppm} \mathrm{Cl}$ in nutrient solution culture. Root osmotic potential seemed not to be related to root $\mathrm{Na}$ and $\mathrm{Cl}$ content.

Osmotic potential of leaves in sand culture was greatly decreased at 500 and $1,000 \mathrm{ppm}$ $\mathrm{Cl}$ showing -14.5 and -15.0 bars, respectively. In nutrient solution culture it also decreased at 4,000 and $5,000 \mathrm{ppm} \mathrm{Cl}$ showing -15.0 and -24.2 bars, respectively. These reductions of leaf osmotic potential were accompanied by visible salt injury symptoms and a reduction of leaf fresh weight. Therefore, the degree of salt injury at $4,000 \mathrm{ppm}$ $\mathrm{Cl}$ in nutrient solution culture was compared to that at $500 \mathrm{ppm} \mathrm{Cl}$ in sand culture. The osmotic potential of the treatment solution was -6.0 bars at $4,000 \mathrm{ppm} \mathrm{Cl}$. That of sand solution must be approximately -6.0 bars, although it was not determined.

Lower transpiration rates of salinity-affected plants have already been reported $(9,10$, $11,14,17)$. Water consumption of salinityaffected plants was considerably lower than that of the control plants and this may be ascribed to lower transpiration rate and smaller transpiration area (14). As shown in Table 9, the decrement of transpiration rate (g. water lost/g. leaf dry weight) was smaller than that of transpiration amount (g. water lost/plant). This could be related to the pressure potential in the leaf tissue which is generally proportinal to the degree of stomatal closure $(7,26)$.

\section{Acknowledgement}

We are thankful to Dr. E. L. Proebsting, Washington State University, for reading this manuscript.

\section{Literature Cited}

1. AYERS, A.D., and H.E. HAYWARD. 1948. A method for measuring the effects of soil salinity on seed germination with observation on several vegetable crop plants. Soil Sci. Soc. Amer. Proc. $13: 224-226$.

2. Ayers, A. D., C. H. WAdLeigh, and L. BerNSTEIN. 1951. Salt tolerance of six varieties of lettuce. Proc. Amer. Soc. Hort. Sci. 57 : $237-242$. 
3 Bernstein, L., and A. D. Ayers. 1951. Salt tolerance of six varieties of green beans. Proc. Amer. Soc. Hort. Sci. 57 : 243-248.

4. CoOper, A. W., and E. B. DUMBrofF. 1973. Plant adjustment to osmotic stress in balanced mineral-nutrient media. Can. J. Bot. $51: 763$ -773 .

5. Francois, L. E., and L. BERnstein. 1964. Salt tolerance of safflower. Agron. J. $56: 38$ -40 .

6. Ehlig, C. F., and L. Bernstein. 1958. Salt tolerance of strawberries. Proc. Amer. Soc. Hort. Sci. 72 : 198-206.

7. GALE, J., H. C. KOHL, and R. M. HAGAN. 1967. Changes in the water balance and photosynthesis of onion, bean and cotton plants under saline conditions. Physiol. Plant. 20 : $408-420$.

8. HAYWARD, H.E., and E. M. LONG. 1941. Anatomical and physiological responses of the tomato to varying concentrations of sodium chloride, sodium sulphate, and nutrient solutions. Bot. Gaz. $102: 437-462$.

9. LagerwerfF, J. V., and H.E. EAgle. 1962. Transpiration related to ion uptake by beans from saline substrates. Soil Sci. 93:420430.

10. Lunin, J., M. H. Gallatin, and A.R. BATCHELDER. 1961. Effect of stage of growth at time of salinization on the growth and chemical composition of beans. II. Salinization in one irrigation compared with gradual salinization. Soil Sci. 92 : 194-201.

11. Lunin, J., M. H. Gallatin, and A. R. BatCHELDER. 1963. Saline irrigation of several vegetable crops at various grow th stages. I. Effect on yields. Agron. J. 55 : 107-110.

12. Masui, M., A. Ishida, A. NukAYA, and T. OGURA. 1976. Studies on the salt tolerance of muskmelons. II. Salt tolerance in sand and nutrient solution cultures. Abstract. 1976 Spring Meeting Japan. Soc. Hort. Sci. 254255. (Japanese)

13. MASUI, M., A. NUkAYA, and A. IshidA. 1975. Salt content of well water of greenhouse growers in Shizuoka Prefecture. Bul. Fac. Agr., Shizuoka Univ. Japan. $25: 15-22$. (Japanese with English summary)
14. Meiri, A., and A. POLJAKOF-MAYBer. 1970. Effect of various salinity regimes on growth, leaf expansion and transpiration rate of bean plants. Soil Sci. 109 : 26-34.

15. NiEman, R.H. 1962. Some effects of sodium chloride on growth, photosynthesis, and respiration of twelve crop plants. Bot. Gaz. 123 : $279-285$.

16. NUKAYA, A., M. Masui, A. Ishida, and T. OGURA. 1977. Salt tolerance of green soybeans. J. Japan. Soc. Hort. Sci. $46: 18$ 25.

17. NUKAYA, A., M. MASUI, and A. IShidA. 1979. Salt tolerance of tomatoes. J. Japan. Soc. Hort. Sci. 48 : 73-81.

18. NUKAYA, A., M. MASUI, and A. ISHIDA. 1980. Salt tolerance of muskmelons grown in different salinity soils. J. Japan. Soc. Hort. Sci. $48: 468-474$.

19. NUKAYA, A. Unpublished data.

20. OSAWA, T. 1960. Studies on the salt tolerance of vegetable crops in sand culture. I. On fruit vegetables. J. Japan. Soc. Hort. Sci. $29: 42-52$. (Japanese with English summary)

21. OSAWA, T. 1961. Ditto $\mathrm{N}$. On the relative salt tolerance and the salt injury of vegetable crops with special reference to the mineral nutrition. Ibid. $30: 241-252$. (Japanese with English summary)

22. Shannon, M. C., and L. E. Francois. 1978. Salt tolerance of three muskmelon cultivars. J. Amer. Soc. Hort. Sci. $103: 127-130$.

23. SHIMADA, N. 1969. Studies on the salt iniury of crops (Part 1). On the root activity of cucumber seedlings in single salt solution. J. Sci. Soil \& Manure, Japan. 40 : 26-31. (Japanese)

24. Shmueli, M., and D. GoldberG. 1971. Sprinkle, furrow and trickle irrigation of muskmelon in an arid zone. Hort Sci. $6: 557-559$.

25. Shourbagy, N.E., and A. WALlACE. 1965. Sodium accumulation and sodium responses of five varieties of barley. Agron. J. $57: 449$ -450 .

26. Slatyer, R. O. 1961. Effects of several osmotic substrates on the water relationships of tomato. Aust. J. Biol. Sci. $14: 519-540$ 。 
砂耕と養液耕におけるメロンの耐塩性

糠 谷 明・増井 正夫・石田 明

(静岡大学農学部)

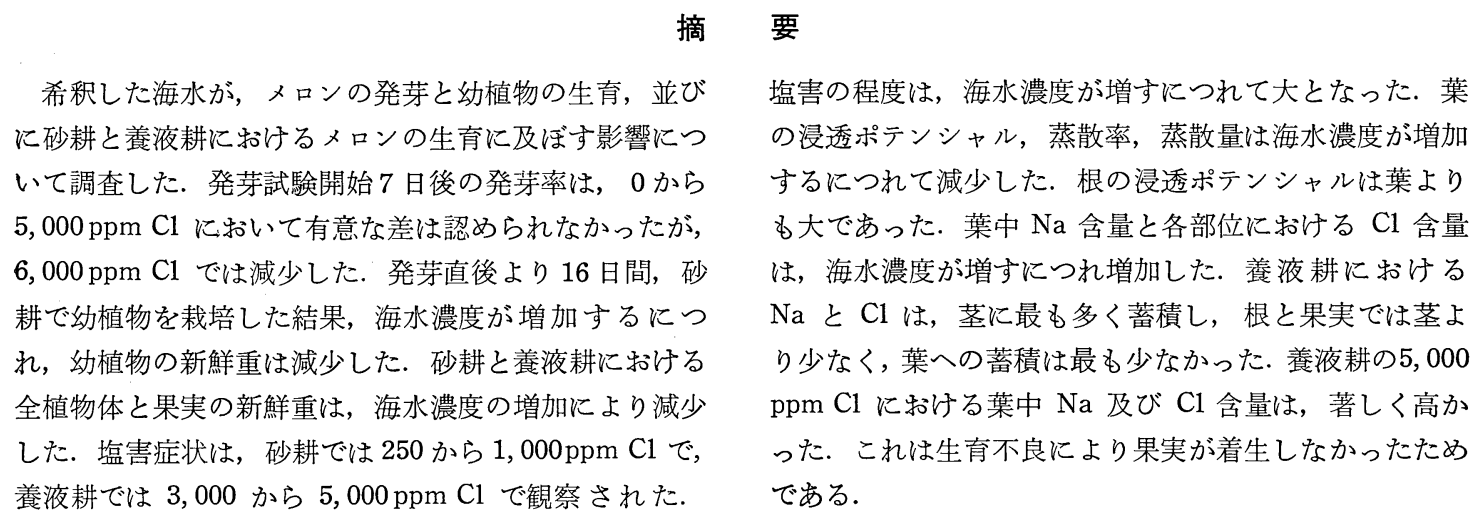

